\title{
Biología floral y polinización de Ipomoea murucoides Roem. \& Schult. (Convolvulaceae) en Ixtapan del Oro, Estado de México (México)
}

Luis Antonio Caballero-Martínez, Luis Isaac Aguilera-Gómez, Irma Victoria Rivas-Manzano, Carlos Jorge AguilarOrtigoza \& Valentina Lamus-Molina

Universidad Autónoma del Estado de México. Facultad de Ciencias, Campus El Cerrillo Piedras Blancas, Carretera Toluca Ixtlahuaca Kilómetro 15.5, Toluca, Estado de México, México.

Correspondencia

L.A. Caballero-Martínez

E-mail: caballero.la@gmail.com

Tel: $\quad+527222965556$

Fax: +52 7222965554

Recibido: 26 julio 2012

Aceptado: 20 noviembre 2012

Publicado on-line: 23 noviembre 2012

\section{Resumen}

En el presente estudio se describen algunas de las adaptaciones de las flores de Ipomoea murucoides con respecto a su interacción con los visitantes florales así como el sistema de reproducción sexual en una Selva Baja Caducifolia de México. La antesis crepuscular, el patrón en la producción de néctar y la concentración de azúcares, el pedicelo robusto y el color y tamaño de las flores son congruentes con el síndrome floral de la quiropterofília. No obstante, se observó que además del murciélago Anoura geoffroyi, las flores de I. murucoides también las visitaron durante el día colibríes (Colibri thalassinus, Eugenes fulgens y Heliomaster constantii), abejas carpinteras (Xylocopa guatemalensis y $X$. tenuata) y escarabajos (Cerambycidae). Los murciélagos, al ser los primeros visitantes de las flores, resultaron ser los polinizadores más importantes de I. murucoides, una especie alógama y, al parecer, autoincompatible.

Palabras clave: Autoincompatibilidad, Éxito reproductor, Polinización, Quiropterofilia.

\begin{abstract}
Floral biology and pollination of Ipomoea murucoides Roem. \& Schult. (Convolvulaceae) in Ixtapan del Oro, State of Mexico (Mexico)

The present study describes some of the Ipomoea murucoides flowers adaptations regarding to floral visitors interactions and sexual reproduction system in a deciduous forest in Mexico. Crepuscular anthesis, nectar production pattern and sugar concentration, robust pedicel, color and flower size are consistent with chiropterophilous floral syndrome. However, it was observed that in addition to the Anoura geoffroyi bats, the I. murucoides flowers are also visited during the day by hummingbirds (Colibri thalassinus, Heliomaster constantii and Eugenes fulgens), carpenter bees (Xylocopa guatemalensis and $X$. tenuata) and beetles (Cerambycidae). The bats, being the first flowers visitors, turned out to be the most important $I$. murucoides pollinators, an apparently self-incompatible and allogamous plant.
\end{abstract}

Key words: Self-incompatibility, Reproductive success, Pollination, Chiropterophily. 


\section{Introducción}

El género Ipomoea Sendtn. ex Vell., formado por un grupo de entre 600 y 1000 especies (Miller et al. 2004), se compone por plantas con flores hermafroditas que muestran gran variación tanto en el momento de la antesis como en la coloración de la corola y en los patrones de secreción de néctar, razón por la cual las especies de Ipomoea poseen una alta diversidad de polinizadores (Matuda 1966, McDonnald 1991). La mayoría de las plantas del género son herbáceas, sin embargo, desde el centro de México hasta Guatemala ha evolucionado un conjunto de 8 a 10 especies con hábitos arborescentes, que además de presentar un tallo leñoso, se caracterizan por que tienen flores blancas, sépalos coriáceos, frutos dehiscentes y semillas con tricomas marginales (Murguía et al. 1995, Carranza et al. 1998, Carranza 2001), por lo que se clasifican dentro de la serie Arborescens (McPherson, 1981). Se conoce poco sobre la ecología floral de estos árboles, se ha descrito que las flores de Ipomoea arborescens Humb. \& Bonpl. ex Willd son visitadas por el murciélago Leptonycteris nivalis Saussure 1860 en Tepoztlán, Morelos, México (Sánchez \& Medellín 2007), que las flores de $I$. wolcottiana Rose son visitadas por esfíngidos, himenópteros y aves en Chamela, Jalisco, México (Parra-Tabla 2002), y que las flores de I. murucoides son frecuentadas por el murciélago nectarívoro Anoura geoffroyi Gray, 1838 en Ixtapan del Oro, Estado de México (Caballero et al. 2007), por lo que se sospecha que posiblemente esta planta presenta el síndrome floral de la quiropterofília. Al momento, tan solo se ha documentado una descripción general sobre I. murucoides, pero se desconocen aspectos biológicos asociados a la reproducción, fenología floral, ritmo de apertura de las flores, patrón de producción y concentración de azúcares del néctar, caracteres florales, visitantes florales y éxito reproductor, siendo estos aspectos los objetivos del trabajo.

I. murucoides se distribuye desde México hasta Guatemala en la vegetación de Selva Baja Caducifolia (Rzedowski 1978), en altitudes que oscilan entre los 700 a 1500 msnm (Matuda 1966, Gentry 1995, Murguía et al. 1995, Carranza et al. 1998, Carranza 2001). Es un árbol con flores hermafroditas, actinomorfas, tetracíclicas y pentámeras, el cáliz es tomentoso, la corola es blanca gamopétala de 6-7 cm, los estambres se encuentran adnados a la corola y la antera presenta dehiscen- cia longitudinal. El ovario es súpero y glabro, los estigmas son globosos y se encuentran capitados o bifidos (Matuda 1966, Rzedowski \& Rzedowski 1985, Carranza \& Murguía 1998), y el polen es radiosimétrico, apolar, esferoidal de 113,8 $\mu \mathrm{m}$, pantoporado con la exina foveolada (Buril et al. 2008; Tovar et al. 2009).

\section{Material y métodos}

\section{Área de Estudio}

El municipio de Ixtapan del Oro se localiza en la porción Occidental del Estado de México. La cabecera municipal se encuentra en $19^{\circ} 15^{\prime} 44^{\prime \prime} \mathrm{N}$, $100^{\circ} 15^{\prime} 50^{\prime}$ ' O, a una altitud de $1,672 \mathrm{msnm}$ (Fig. 1). El clima es semicálido subhúmedo con lluvias en verano $(\mathrm{A}) \mathrm{c}(\mathrm{W}) \mathrm{a}(\mathrm{i}) \mathrm{g}$. La vegetación que en las partes altas se desarrolla es el Bosque de PinoEncino y en las partes bajas de la cañada predomina la Selva Baja Caducifolia (Miranda \& Hernández 1963, Álvarez \& Álvarez-Castañeda 1996). La población de I. murucoides estudiada se encuentra predominantemente en el área correspondiente a la Selva Baja Caducifolia con una densidad de 46 individuos reproductores por hectárea.

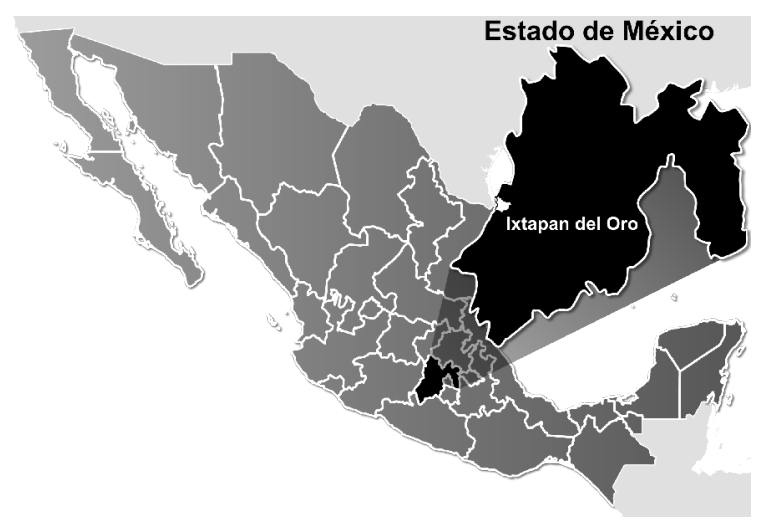

Figura 1. Ubicación geográfica del municipio de Ixtapan del Oro. Figure 1. Geographic location of the Ixtapan del Oro municipality.

\section{Selección de la muestra}

Se estableció la época de floración de I. murucoides por medio de visitas quincenales desde marzo de 2009 hasta marzo de 2010. Al mismo tiempo, se seleccionaron marcándolos con cintas adhesivas de colores, diez árboles por el método del vecino más cercano (Fix \& Hodges 1951), con una adaptación que consistió en medir la distancia del punto al árbol de I. murucoides más cercano y posteriormente se ubicó el vecino más contiguo de 
la misma especie para tener en la muestra sólo individuos de I. murucoides, se calculó la densidad de individuos por hectárea y con la finalidad de describir el pico de floración, se realizaron nueve conteos quincenales de las flores producidas en los diez individuos marcados. Los ejemplares recolectados se depositaron en el Herbario de la Facultad de Ciencias de la Universidad Autónoma del Estado de México.

\section{Biología floral}

Se realizó la caracterización morfométrica de cien flores en antesis de 21 individuos. Se midió, con un calibrador mecánico, la longitud de las flores, la longitud interna, el diámetro de la flor, el diámetro interno de la corola, la distancia de la antera y del estigma a la base del tubo de la corola y el diámetro del pedicelo (Fig. 2).

Se estableció en diez flores, de individuos diferentes, el tiempo en que acontece la antesis y la receptividad considerando la turgencia del estilo y los filamentos estaminales, se estableció el olor de las flores por medio de la percepción humana, en donde se identificó si el olor era simpático o idiopático. También, empleando la fórmula del volu-

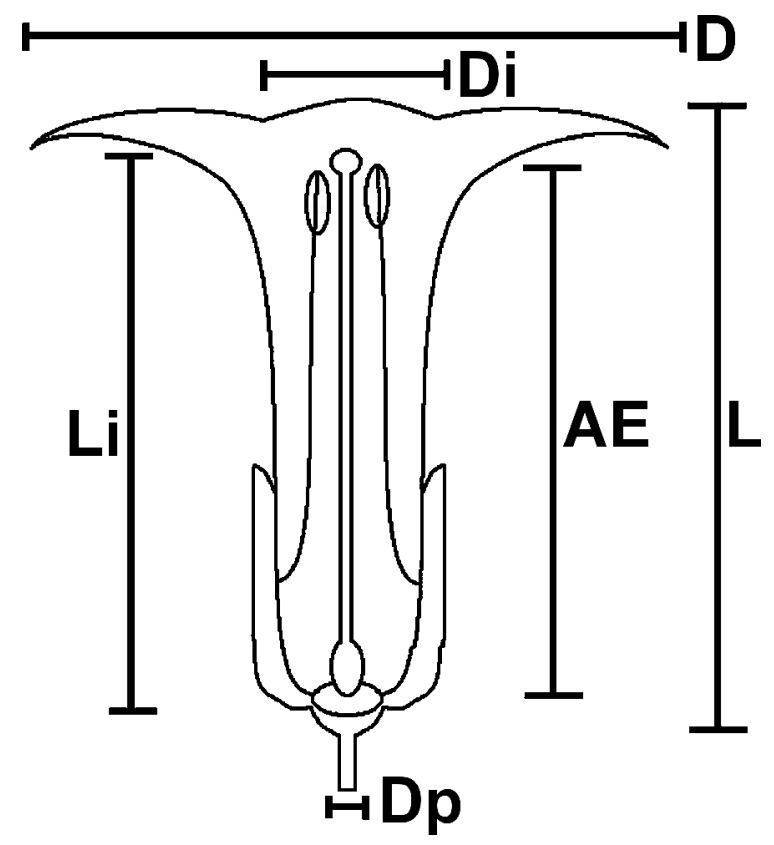

Figura 2. Medidas consideradas en las flores de Ipomoea murucoides; longitud de la flor (L), longitud interna (Li), diámetro de la flor (Df), diámetro de la apertura interna de la corola (Di), distancia de la antera y del estigma a la base del tubo floral (AE) y diámetro del pedicelo (Dp).

Figure 2. Measures considered in the Ipomoea murucoides flowers; flower length (L), internal length (Li), flower diameter (Df), internal diameter (Di), distance from the anther and stigma to the floral tube basis (AE) and pedicel diameter (Dp). men del cilindro $\left(v=\pi \cdot r^{2} \cdot h\right)$, en diez flores cubiertas de árboles distintos se midió con tubos capilares (de $75 \mathrm{~mm}$ de longitud y 1,4 $\mathrm{mm}$ de diámetro), el volumen de néctar cada dos h, así como la concentración de azúcares en esos intervalos de tiempo con un refractómetro Atago HSR-500 según lo indicado por Ibarra y colaboradores (2005), en cada censo se consideró a la misma flor y se realizó una ANOVA unidireccional y la prueba de diferencia significativa honesta (DSH) de Tukey para agrupar la información de las medias de la disponibilidad de néctar y la concentración de azúcares por cada hora de muestreo.

\section{Determinación de los visitantes florales}

Para conocer a los visitantes florales nocturnos se consideraron los síndromes florales de la quiropterofilia y de la falenofília al seleccionar a diez flores de los diez árboles marcados que estaban próximas a la antesis, y bien expuestas para permitir la instalación de cámaras de video (Muchhala 2006). Estas flores se filmaron semanalmente durante 10 días con una videocámara Sony DCRSR45 con sistema de visión nocturna que se ubicó sobre un trípode a una distancia aproximada de dos metros de las flores. Se filmó a cada flor durante $5 \mathrm{~h}$, desde las 20:00 a las 1:00 h y se obtuvieron en total $50 \mathrm{~h}$ de filmación por todas las flores. En los diez árboles marcados también se seleccionaron otras 10 flores próximas a la antesis que se encontraran expuestas y agregadas debido a que los insectos y las aves tienden a visitar flores con estas características y también se consideró la idea del individuo más vigoroso al seleccionar las flores (Rocha et al. 2005). En estas flores se realizaron seguimientos semanales con binoculares durante 10 días de las 6:00 a las 19:00 h en cada flor, y resultaron aproximadamente $120 \mathrm{~h}$ de vigilancia al término de la investigación. En los videos y en los seguimientos se identificaron como visitantes florales importantes a los animales que tocaron el androceo y el gineceo y se cuantificó el número de visitas que realizaron por hora así como su duración. Las aves se determinaron mediante las guías de identificación de campo de Howell \& Webb (1995) y de Peterson \& Chalif (2008) y se contrastó su presencia en la zona con los trabajos de Navarijo \& Neri (2000), Bojorges (2004) y Canales \& Altamirano (2004). Los insectos se capturaron cuando visitaban las flores de $I$. murucoides con una red entomológica, y se enviaron a taxónomos expertos de la Universidad $\mathrm{Na}$ - 
cional Autónoma de México para su determinación, estos ejemplares se depositaron en la colección de insectos de la Facultad de Ciencias de la misma universidad.

\section{Especificidad de los quirópteros con $I$. murucoides}

En los meses de floración de I. murucoides, quincenalmente se realizaron siete trampeos nocturnos de quirópteros de las 21:00 a las 2:00 h con una red de niebla de 2,5 X 6 m colocada cerca de los árboles marcados. Al liberar los murciélagos de las redes, se les determinó a nivel de especie con la clave de Medellín et al. (1997) y en caso de pertenecer a la subfamilia Glossophaginae, se colectaron los residuos depositados en la superficie del pelaje con el fijador gelatina de Kisser (Fasola et al. 2005, Caballero-Martínez et al. 2009); se colocó a los murciélagos en una bolsa de manta por espacio de una hora para obtener muestras de heces y más tarde fueron liberados (Dinerstein 1986, Stockwell 2001). Las muestras de los residuos se prepararon en laminillas, se les observó en un microscopio compuesto de campo claro en un aumento de 400X. Se estableció la presencia de polen, se determinaron las especies de plantas a las que éste corresponde y se evaluó la frecuencia de las especies encontradas en las muestras (Herrera \& Martínez del Río 1998, Muchhala \& Jarrín-V 2002, Caballero-Martínez et al. 2009). Las muestras de las heces de los murciélagos se montaron en laminillas con gelatina de Kisser y en un microscopio se determinaron las especies de las plantas a las que corresponden los granos de polen por comparación con la colección de polen de referencia de la flora local (Caballero-Martínez 2007), y por medio de los trabajos palinológicos que tratan especies mexicanas (Palacios-Chávez et al. 1992, Telleria \& Daners 2003, Caballero-Martínez et al. 2009, Tovar et al. 2009), y se evaluó su frecuencia (Kunz \&Whitaker 1983, CaballeroMartínez et al. 2009). Finalmente, se determinó el grado de especificidad de los murciélagos con $I$. murucoides considerando la frecuencia de cada especie de planta identificada en las muestras (Ibarra et al. 2005).

\section{Calidad de los visitantes florales}

Para reconocer a un animal como el principal visitante floral se consideran dos cualidades: la cantidad y la calidad. La cantidad se refiere al número de polen conespecífico que puede depositar sobre el estigma, mientras que la calidad del vector se manifiesta en el grado de especificidad con una planta (Muchhala et al. 2008). Por lo tanto, para comprobar la calidad de los visitantes florales de I. murucoides, en diez flores de los diez árboles de la muestra seleccionada se envolvieron las partes sexuales de la flor con una cinta adhesiva transparente para colectar el polen depositado. Se utilizaron dos cintas por cada flor, una para los visitantes florales diurnos y otra para los nocturnos. Al desmontar las cintas adhesivas se frotaron con el fijador gelatina de Kisser y se montaron en laminillas. Posteriormente en el microscopio, se identificaron los granos de polen de I. murucoides (Tovar et al. 2009) y el resto se clasificó como polen heteroespecífico y se cuantificaron por la técnica de escaneo (Girón 1995, Caballero-Martínez et al. 2009). Por último se calculó el porcentaje del total de polen conespecífico y el heteroespecífico en las muestras (Muchala 2006), y se aplicó una prueba de Chi-Cuadrado (Zar 1996, Muchhala \& Jarrín-V 2002), para conocer la diferencia en la contribución de los polinizadores diurnos y los nocturnos en el transporte de polen de I. murucoides y de otras especies (Ibarra et al. 2005).

Con el objetivo de conocer si las flores de $I$. murucoides eran autocompatibles y la contribución de los polinizadores diurnos y nocturnos en la producción de frutos y semillas se aplicó el procedimiento propuesto por Ibarra et al. (2005). Durante la época de floración se aplicaron cinco tratamientos en días consecutivos en una muestra de cien flores próximas a la antesis pertenecientes a los diez individuos previamente marcados, se consideraron veinte flores en total para cada procedimiento (dos flores de cada uno de los diez árboles seleccionados por experimento). En el primer tratamiento se embolsaron las veinte flores con tela mosquitera para impedir el paso de los polinizadores y observar si era posible la autopolinización. En el segundo tratamiento se observó si la autopolinización es posible aunque no ocurra de forma natural, para lo cual, se cubrieron las veinte flores y se indujo la autopolinización al trasladar polen de las anteras al estigma de una misma flor. En el tercer tratamiento se probó la efectividad de los polinizadores nocturnos, en donde se dejaron descubiertas las veinte flores durante la noche para permitir el paso de los visitantes florales nocturnos, posteriormente a partir de las 6:00 h se encerraron las flores. En el cuarto tratamiento en otras veinte flores de estos árboles, se examinó la efec- 
tividad de los polinizadores diurnos al cubrir las flores desde la antesis durante la noche y por la mañana a las 6:00 h se les destapó; el quinto tratamiento consistió en el grupo control o de polinización libre. Un mes después, se cuantificó por cada tratamiento el número de flores abortadas, la cantidad de frutos producidos y se sumaron las semillas formadas por cada fruto. Posteriormente, se calculó el éxito reproductivo preemergente, siguiendo a Wiens et al. (1987), según la siguiente ecuación: [(número de frutos obtenidos / número de flores tratadas) x (número promedio de semillas obtenidas por fruto / número promedio de óvulos por flor)], que en definitiva mide el $\mathrm{n}^{\circ} \mathrm{de}$ óvulos que se transforman en semillas en los distintos tratamientos. Se realizó la Prueba de Probabilidad Exacta de Fisher (Zar 1996, Muchhala \& Jarrin-V 2002), para conocer las posibles diferencias de la producción de frutos en las flores de los tratamientos para polinizadores diurnos y los nocturnos.

\section{Resultados}

\section{Fenología floral y duración de las flores en antesis}

En el ciclo de muestreo de noviembre de 2009 a febrero de 2010 (Fig. 3), la época de floración de I. murucoides ocurrió desde la última semana del mes de noviembre hasta la primera semana de febrero y tuvo una duración aproximada de 12 semanas y se registró un promedio de 68,1 flores por árbol (d.s. $=56,33 ; n=10)$. Se presentó el mayor número de flores durante la segunda quincena del mes de diciembre con un total de 247 flores y se obtuvo un promedio de 24,7 flores por individuo (d.s. $=21,52 ; n=10)$.

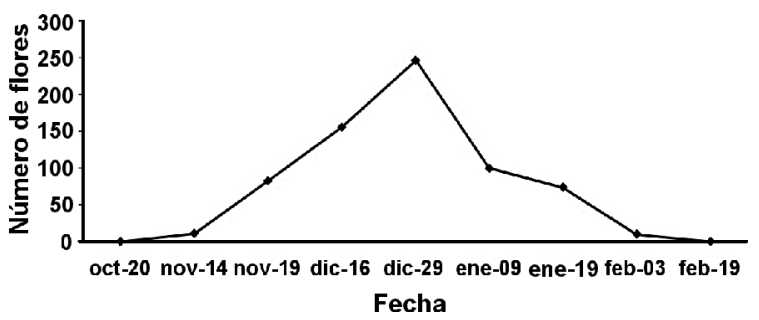

Figura 3. Fenología floral de Ipomoea murucoides $(\mathrm{n}=10)$ durante los días de muestreo de octubre a febrero. En la abscisa se muestra el número de flores y en la ordenada las fechas de muestreo.

Figure 3. Floral phenology of Ipomoea murucoides $(\mathrm{n}=10)$ during the sampling days from October to February. The abscissa shows the flowers number and the ordinate the sampling dates.
La antesis de las flores aconteció por la tarde a partir de las 16:00 h, se mantuvo la turgencia de las estructuras reproductivas durante casi todo el tiempo en que permanecieron abiertas $(n=10)$. Las flores muestreadas $(\mathrm{n}=100)$ presentaron un diámetro medio del pedicelo de $0,521 \mathrm{~cm}$ y la corola blanca, con un diámetro medio de $6,94 \mathrm{~cm}$, una longitud promedio de $5,9 \mathrm{~cm}$ con apertura interna de la corola de $2 \mathrm{~cm}$ de diámetro (Tabla 1). El olor fue tenue e idiopático, semejante al del ajo $(\mathrm{n}=10)$ y los ovarios presentaron 4 óvulos $(\mathrm{n}=21)$.

\begin{tabular}{lcc}
\hline \multicolumn{1}{c}{ Característica Floral } & $\begin{array}{c}\text { Promedio } \\
(\mathrm{cm})\end{array}$ & $\begin{array}{c}\text { Desviación } \\
\text { Estándar }\end{array}$ \\
\hline Longitud de la flor & 5,898 & 0,628 \\
Longitud interna & 4,609 & 0,536 \\
$\begin{array}{l}\text { Diámetro de la flor } \\
\begin{array}{l}\text { Diámetro de la apertura } \\
\text { interna de la corola }\end{array}\end{array}$ & 6,939 & 0,775 \\
$\begin{array}{l}\text { Distancia de la antera a la } \\
\text { base del tubo floral }\end{array}$ & 1,938 & 0,257 \\
$\begin{array}{l}\text { Distancia del estigma a la } \\
\text { base del tubo floral }\end{array}$ & 4,859 & 0,561 \\
Diámetro del pedicelo & 4,992 & 0,756 \\
\hline
\end{tabular}

Tabla 1. Se describen los promedios y la desviación estándar de las medidas de 100 flores de 21 individuos de Ipomoea murucoides. Las flores presentaron una longitud máxima de 7,36 y una mínima de $4,16 \mathrm{~cm}$.

Table 1. Describes the measures average and the standard deviation of 100 flowers of 21 individuals of Ipomoea murucoides. The flowers had a maximum length of 7.36 and a minimum of $4.16 \mathrm{~cm}$.

\section{Producción de néctar de las flores y concentración de azúcares}

El máximo valor de producción de néctar de las flores se registró a las 23:00 h con 55,5 $\mu \mathrm{l}$ (d.s. $=9,7 ; \mathrm{n}=10$ ), y el mínimo a las 15:00 h con $11,6 \mu \mathrm{l}$ (d.s. $=5,6 ; \mathrm{n}=10)$. La mayor concentración de azúcares se presentó a las 23:00 h con 26,1 ${ }^{\circ}$ Brix (d.s. $=0,31 ; \mathrm{n}=10$ ), posteriormente, disminuyó la concentración y se mantuvo fluctuando alrededor de los $21^{\circ}$ Brix (Fig. 4). La prueba de diferencia significativa honesta (DSH) de Tukey indicó que existieron diferencias significativas entre los valores de las medias de la disponibilidad de néctar y la concentración de azúcares entre las mediciones del día y de la noche.

\section{Visitantes florales y eficacia polinizadora}

Se identificaron tres clases de visitantes florales nocturnos (mamíferos) y diurnos (aves e insectos). 


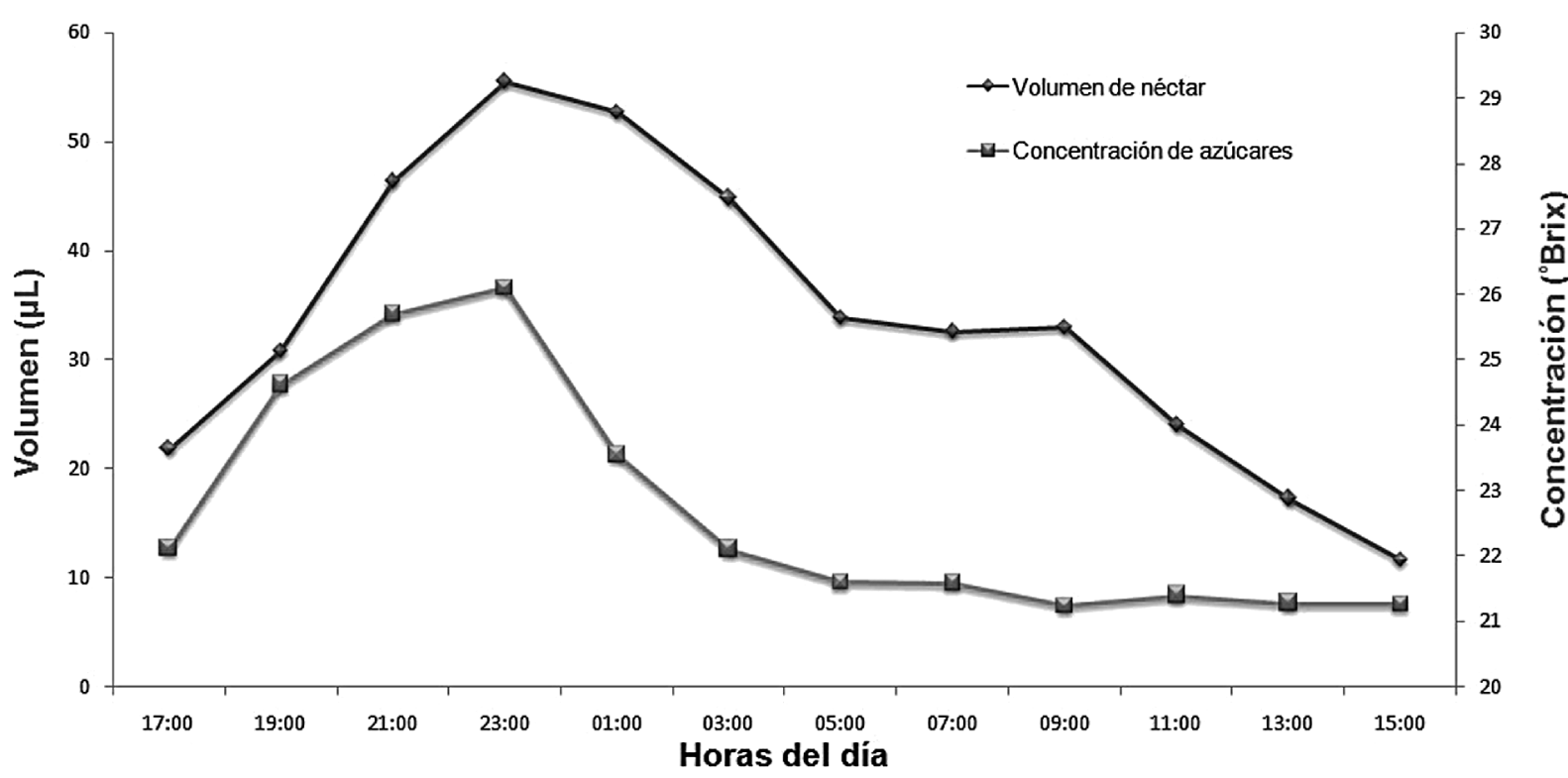

Figura 4. Promedio de la producción de néctar $(\mu \mathrm{L})$ y de la concentración de azúcares ( ${ }^{\circ}$ Brix $)$ en Ipomoea murucoides $(\mathrm{n}=10)$ por cada dos horas durante el día.

Figure 4. Average of the nectar production (ul) and the sugar concentration ( ${ }^{\circ}$ Brix) in Ipomoea murucoides $(\mathrm{n}=10)$ for every two hours during the day.

\section{Mamíferos}

El mamífero que visitó las flores de 1 . murucoides es el quiróptero A. geoffroyi (familia Phyllostomidae). En los videos se verificó que la mayor actividad de estos animales en las plantas de I. murucoides fue de las 21:00 a las 24:00 h. Se observó que los murciélagos introducen el rostro por la apertura de la corola a una alta velocidad por lo que ocasionan en la flor un movimiento brusco. Los quirópteros contactan con la región cefálica el androceo y el gineceo y se impregnan de polen en el pelo del rostro, las orejas y la cabeza. Las visitas que realizaron los murciélagos en las flores duraron alrededor de un segundo $(n=7)$.

Durante la época de floración se capturaron 28 quirópteros, todos de la especie $A$. geoffroyi. En las muestras del pelaje y las heces de estos murciélagos se reconocieron tres tipos de polen: $I$. murucoides, Agave sp. L. y Asteraceae (Tabla 2).

\section{Aves}

La mayoría de las aves que interactuaron con las flores de I. murucoides pertenecen a la familia Trochilidae y se identificaron tres especies: Colibri thalassinus Swainson, 1827, Eugenes fulgens Swainson, 1827 y Heliomaster constantii De Lattre, 1843. Los colibríes comenzaron la actividad como visitantes florales de $I$. murucoides a partir de las 6:00 h y terminaron por la tarde después de las 19:00 h. Los colibríes permanecieron en vuelo suspendido frente a las flores por un promedio de 4,23 segundos (d.s. $=0,78$ seg.; $n=43$ ). Tocaron con el pico y la región cefálica el estigma y las anteras y en el caso de H.constantii se notó que visitaron flores abiertas y cerradas.

\begin{tabular}{lccc}
\multicolumn{1}{c}{ Especie } & Polen & Porcentaje & $\begin{array}{c}\text { Frecuencia } \\
\text { (\%) }\end{array}$ \\
\hline $\begin{array}{l}\text { lpomoea } \\
\text { murucoides }\end{array}$ & 6014 & 99,85 & 100 \\
Agave sp. & 5 & 0,083 & 17,9 \\
Asteraceae sp. & 4 & 0,066 & 7 \\
Total & 6023 & 100 & \\
\hline
\end{tabular}

Tabla 2. Total de granos de polen y su porcentaje en las muestras del pelaje y de las heces de Anoura geoffroyi $(\mathrm{N}=28)$ durante la época de floración de Ipomoea murucoides.

Table 2. Total pollen grains and their percentage in the Anoura geoffroyi fur and feces samples $(\mathrm{N}=28)$ during the flowering season of Ipomoea murucoides.

\section{Insectos}

Fueron diversos los insectos que visitaron las flores de I. murucoides. Los formícidos visitaron estas plantas para cortar las hojas. La base de la corola sirve de habitáculo para dermápteros y coleópteros pequeños, los cuales no se observó que tocaran las estructuras reproductivas florales y generalmente se encontraron dentro de las flores marchitas y cerradas. Se observó una visita diurna (11:00 h) de un escarabajo de la familia Cerambycidae, este insecto se posó sobre una flor por espacio de 6,32 minutos $(\mathrm{n}=1)$ y su región ventral del tórax, el abdomen, la porción cefálica y las ante- 
nas palparon las anteras y el estigma.

Los lepidópteros diurnos no se acercaron a las flores de I. murucoides, sin embargo, en tres ocasiones polillas de la familia Noctuidae, por las noches (de 21:00 a 24:00 h), se introdujeron en las flores caminando sobre los pétalos y permanecieron en la base de la corola por un período de diez minutos. En los tres casos observados, no se detectó en el video que los noctuidos tocaran las anteras o el estigma con alguna superficie de su

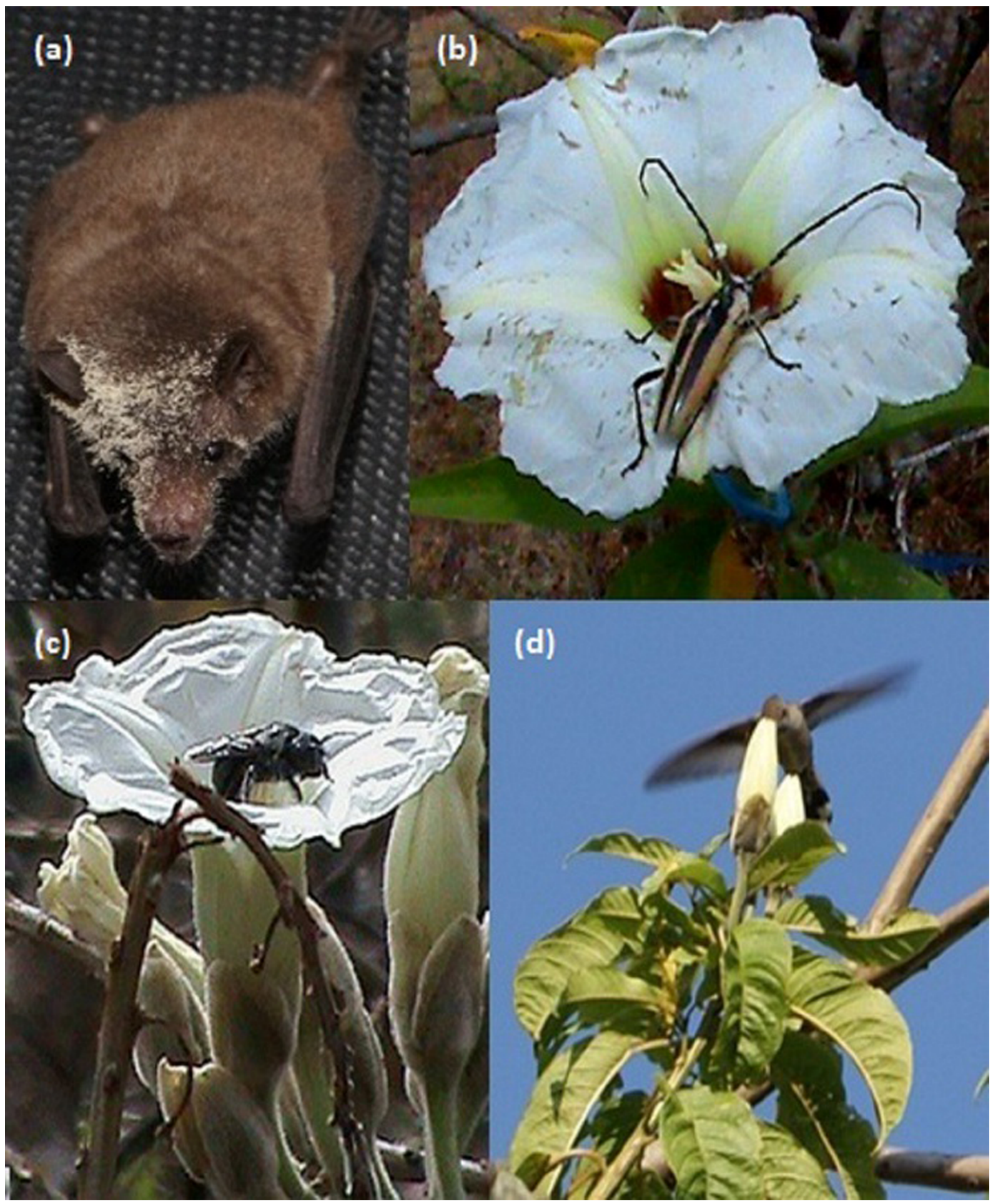

Figura 5. Visitantes florales de Ipomoea murucoides. a: Anoura geoffroyi con polen de I. murucoides; b: Cerambycidae; c: Xylocopa sp.; d: Heliomaster constantii.

Figure 5. Ipomoea murucoides floral visitors. a: Anoura geoffroyi with I. murucoides pollen; b: Cerambycidae; c: Xylocopa sp.; d: Heliomaster constantii. 
cuerpo o las alas y en las observaciones bajo el microscopio tampoco se tienen datos de la presencia de rastros de polen de I. murucoides sobre las polillas.

Fueron varias las especies pertenecientes al orden Hymenoptera que visitaron las flores de $I$. murucoides, entre las abejas lo hicieron las especies Apis mellifera Linnaeus, 1758, Centris sp. Fabricius 1804, Megachile sp. Latreille, 1802, y Lasioglossum sp. Curtis, 1833. Se posaron sobre los pétalos y externamente por la base de la corola sin tocar las anteras ni el estigma, fueron muy pocas las visitas de las abejas a las flores de I. murucoides dado que sólo se observaron a 5 individuos de las 8:00 a las 13:00 h. De estos himenópteros, los que principalmente visitaron las flores de $I$. murucoides fueron dos especies de abejas carpinteras de la familia Apidae, pertenecientes al género Xylocopa Latreille, 1802: X. guatemalensis Cockerell, 1912 y X. tenuata Smith, 1874. Las abejas carpinteras se posaron por un tiempo promedio de 6,09 segundos (d.s.=2,36 seg.; $\mathrm{n}=22$ ) sobre las flores y contactaron el androceo y el gineceo con la región ventral del tórax y el abdomen (Fig. 5).

Respecto a la actividad de los visitantes florales diurnos que tocaron el androceo y el gineceo de I. murucoides, fueron los colibríes (Trochilidae) los que visitaron las flores con mayor frecuencia durante la mañana entre las 7:00 y las
12:00 h y al atardecer entre las 17:00 y las 18:00 h (Fig. 6).

\section{Calidad de los visitantes florales}

Se presentaron diferencias significativas entre las muestras nocturnas y diurnas para el polen conespecífico $\left(X^{2}=652,7 ; \mathrm{gl}=1, \mathrm{p}<0.001\right)$ y para el polen heteroespecífico $\left(X^{2}=4,77 ; \mathrm{gl}=1, \mathrm{p}<0,029\right)$; (Tabla 3).

\section{Sistema de cruzamiento}

En los tratamientos se obtuvieron valores muy bajos de éxito reproductivo en las modalidades de autofecundación espontánea (natural) y manual (artificial) que contrastan con los altos valores observados en la contribución de los visitantes diurnos y nocturnos en la polinización (Tabla 4). No existieron diferencias significativas en la producción de frutos entre los tratamientos de polinización nocturna y diurna $(\mathrm{p}=0,605)$.

\begin{tabular}{lcccc}
\hline \multicolumn{1}{c}{ Tratamiento } & $\begin{array}{c}\text { Flores } \\
(\mathrm{n})\end{array}$ & Frutos & Semillas & Éxito \\
\hline Autopolinización natural & 20 & 1 & 4 & 0,05 \\
Autopolinización artificial & 20 & 2 & 8 & 0,1 \\
Polinización diurna & 20 & 19 & 76 & 0,95 \\
Polinización nocturna & 20 & 17 & 68 & 0,85 \\
Control & 20 & 18 & 72 & 0,9 \\
\hline
\end{tabular}

Tabla 4. Éxito reproductivo de los tratamientos de polinización.

Table 4. Reproductive success of pollination treatments.

\section{Actividad de los visitantes florales diurnos}

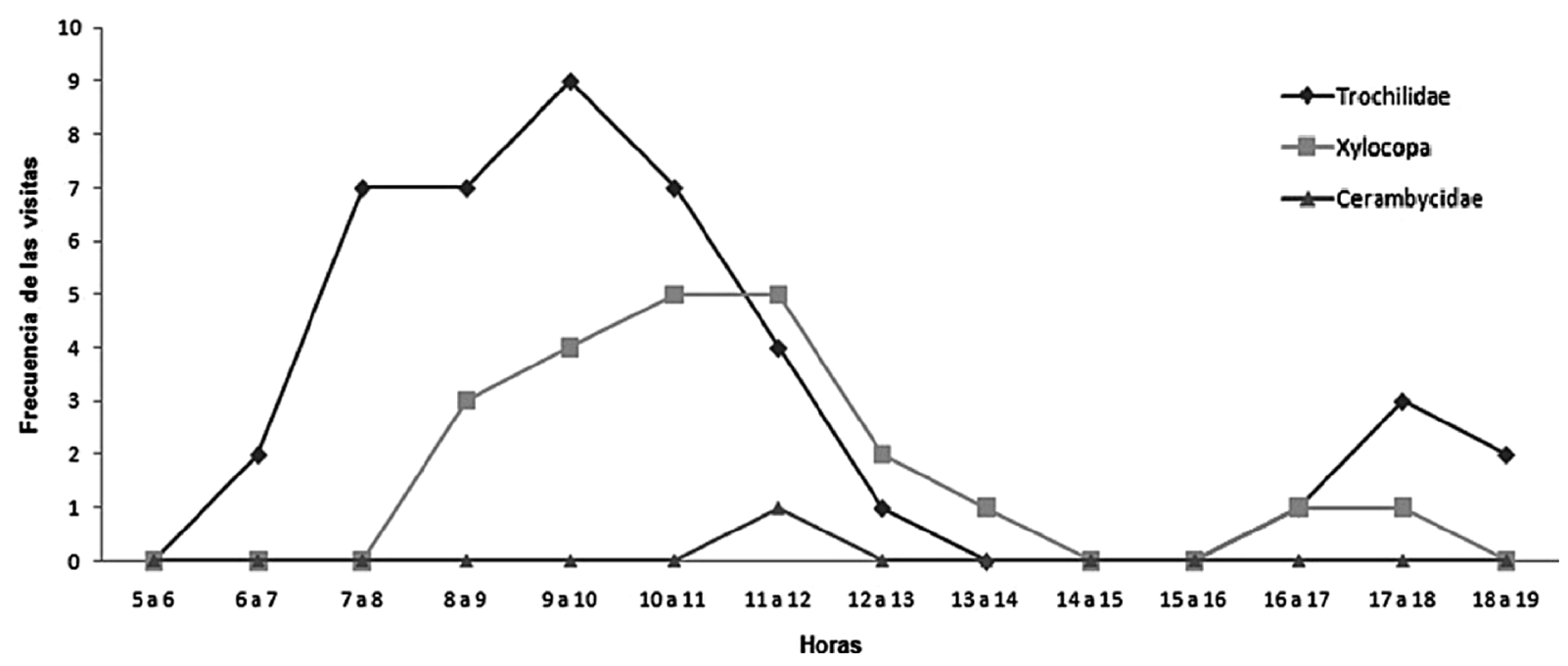

Figura 6. Frecuencias de las visitas a las flores de I. murucoides $(\mathrm{n}=10)$ de los colibríes (Troquilidae), abejas carpinteras (Xylocopa sp.) y escarabajos (Cerambycidae) durante las horas del día.

Figure 6. Frequency of the visits to the I. murucoides flowers $(\mathrm{n}=10)$ of hummingbirds (Troquilidae), carpenter bees (Xylocopa sp.) and beetles (Cerambycidae) during the daylight hours. 


\section{Discusión}

La antesis crepuscular de las flores de I. murucoides a partir de las 16:00 h y el hecho de que el volumen y la concentración de azúcares del néctar alcanzan su máximo valor durante la noche después de las 21:00 h y disminuyen después de las 23:00 h, son indicios de que las flores de esta planta se encuentran especializadas para atraer principalmente a polinizadores nocturnos (Tschapka \& Dressler 2002, Ibarra et al. 2005, Waser 2005). El color blanco de la corola de I. murucoides se relaciona con los síndromes florales de la quiropterofilia y la falenofilia, sin embargo, el olor idiopático de sus flores se encuentra asociado al síndrome floral de la quiropterofília (Janzen 1975, Faegri \& van der Pijl 1979, Proctor et al. 1996, Tschapka \& Dressler 2002, Ibarra et al. 2005, Waser 2005, Muchhala 2006). Las plantas que presentan una apertura de la corola ancha como en el caso de I. murucoides de casi $2 \mathrm{~cm}$ de diámetro son mejor polinizadas por los murciélagos, al permitir el paso de los hocicos alargados de estos animales. Por el contrario, aquellas flores que tienen una apertura de la corola estrecha son eficazmente polinizadas por los colibríes porque guían de mejor forma, el pico hacia el interior, por lo que un mal ajuste en el ángulo de entrada a las flores puede derivar en una disminución de la efectividad en la transferencia de polen (Schemske \& Bradshaw 1999, Muchhala 2007). El pedicelo grueso de $5 \mathrm{~mm}$ de diámetro de las flores de $I$. murucoides puede soportar el peso de la flor y evita que se rompa ante la fuerte envestida de los murciélagos nectarívoros, en Ixtapan del Oro se documentó que los quirópteros $A$. geoffroyi frecuentan las flores de I. murucoides, los adultos de estos murciélagos pesan en promedio $17,47 \mathrm{~g}(\mathrm{Ca}-$ ballero-Martínez et al. 2009), por lo que una flor con un pedicelo frágil se podría romper con la visita de un polinizador de estas dimensiones (Muchhala 2006).

Los visitantes florales que contactan con mayor frecuencia el androceo y el gineceo de las flores de I. murucoides son el murciélago A. geoffroyi, las aves de la familia Trochilidae y las abejas carpinteras del género Xylocopa, por lo que se podrían considerar como los posibles polinizadores de este árbol en Ixtapan del Oro, Estado de México. Durante los meses de noviembre a febrero, A. geoffroyi visita casi de forma exclusiva y con alta frecuencia las flores de I. murucoides, por lo que en la época de floración de esta planta es un visitante floral monoleico. Durante los meses en que $I$. murucoides no florece, $A$. geoffroyi frecuenta a más de ocho especies de plantas a lo largo del año (Caballero et al. 2009). Los murciélagos representan ventajas como polinizadores, ya que debido a sus dimensiones corporales y a la presencia de pelo, son capaces de transportar grandes cantidades de polen y cuando forrajean recorren grandes distancias (Arita \& Martínez del Río 1990). El alto valor de ARI (Índice de relación de aspecto de la longitud y el ancho de las alas), y las alas grandes y delgadas de A. geoffro$y i$, indican que este murciélago puede volar más rápido $\mathrm{y}$, tal vez, recorrer mayores distancias que otros glosofáginos como Glossophaga soricina Pallas, 1766 y Leptonycteris curasoae Miller, 1900 (Tschapka 2004, Sperr et al. 2011). Esta condición incrementa la posibilidad del traslado del polen a largas distancias, el alejarse más del árbol del cual el murciélago toma originalmente el polen, disminuye la probabilidad de encontrar otro individuo de I. murucoides emparentado, por lo que un polinizador con una mayor capacidad de desplazamiento puede favorecer la xenogamia. Los troquílidos que visitan los árboles de I. murucoides generalmente frecuentan flores abiertas pero en ocasiones, algunos individuos de $H$. constantii que tienen un pico recto, penetran por la apertura de la corola en las flores erguidas que permanecen cerradas (Abad-Ibarra et al. 2008). En estos casos, es posible que los colibríes actúen como robadores de néctar, como ya se ha observado en otras especies de plantas (Rojas-Nossa 2007). Las abejas carpinteras del género Xylocopa son generalistas en su forrajeo y tienden a polinizar plantas con altas densidades de flores como $I$. wolcottiana (Parra-Tabla \& Bullock 2002, Loyola et al. 2007). En I. murucoides se observó que los Xylocopa se posan sobre el estigma y las anteras y al retirarse de las flores llevan el abdomen, el dorso y las patas cubiertas de polen, lo que los convierte en polinizadores potenciales. La longitud de más de tres centímetros del androceo y el gineceo de las flores de los árboles de Ipomoea (Matuda 1966, Murguía et al. 1995, Carranza \& Murguía 1998) y la corta probóscide de los Xylocopa de $6,5 \mathrm{~mm}$ en promedio (Navarro y Medel 2009; Potgieter et al., 2009), son limitantes para que las abejas carpinteras puedan alcanzar el néctar cuando se posan sobre los órganos reproductivos de estas flores, por lo que es probable que sólo las vi- 
siten para colectar polen. Las plantas polinizadas por los himenópteros de grandes dimensiones se benefician por el mayor volumen corporal de estos insectos y su abundancia respecto a otros polinizadores, por lo tanto, es importante estudiar aspectos de los Xylocopa como los hábitos de nidificación, sus hábitats, los patrones de vuelo y el comportamiento de forrajeo, para definir con claridad su potencial como polinizadores (NatesParra 2005). Los coleópteros de la familia Cerambycidae no son considerados polinizadores muy eficaces debido a que consumen el polen y su aparato bucal triturador rompe las anteras, pero al ser abundantes adquieren importancia en hábitats en donde son escasos otros polinizadores (Dieringer et al. 1999, Goldblatt et al. 2009). Es posible que los coleópteros Cerambycidae visiten ocasionalmente las flores de I. murucoides pero por la baja frecuencia en la que se les observó sobre las flores no se les puede considerar como polinizadores habituales de estas plantas. Las mariposas nocturnas probablemente son atraídas por el color blanco y olor de las flores de I. murucoides (Matuda 1966), como se ha descrito en el síndrome floral de la falenofilia (Faegri \& van der Pijl 1979, Proctor et al. 1996). Pese a que no tocan el androceo ni el gineceo, las mariposas nocturnas que interactúan con I. murucoides podrían ser importantes para esta planta, las polillas posiblemente actúan como un atrayente secundario para los murciélagos, como se ha comprobado en un estudio realizado por Caballero-Martínez et al. (2009), donde se describe que $A$. geoffroyi consume lepidópteros con alta frecuencia en Ixtapan del Oro.

No obstante, todas las adaptaciones del síndrome floral de la quiropterofília en las flores de $I$. murucoides, es un hecho que en Ixtapan del Oro, Estado de México, sus flores permanecen abiertas durante todo el día con los órganos reproductivos receptivos y contienen polen y néctar con una concentración de azúcares mayor a los $20^{\circ}$ Brix por lo que atraen a visitantes florales diurnos como los insectos y las aves que presentan igual éxito que los murciélagos en su contribución para la producción de frutos. Estas adaptaciones son habilidades que posiblemente contribuyen a la polinización en condiciones en donde los quirópteros nectarívoros son poco abundantes. Álvarez \& Álvarez-Castañeda (1996) encontraron tres especies de murciélagos nectarívoros en esta zona: A. geoffroyi, G. soricina y L. curasoae, sin embargo, al igual que Caballero (2007), sólo fue viable captu- rar a individuos de A. geoffroyi. A pesar de que las plantas polinizadas por los murciélagos son altamente especializadas para atraerlos, existen algunas plantas quiropterofílicas que son polinizadas por otros animales, estos casos tienden a ocurrir en los hábitats en donde la cantidad y la diversidad de los murciélagos nectarívoros es muy baja (Muchhala et al. 2008).

Como otras especies de Ipomoea, por ejemplo Ipomoea trifida Kunth (Kowyama et al. 1994, Kakeda et al. 2000, Kowyama et al. 2000), I. murucoides es posiblemente autoincompatible, ya que solo se obtuvieron 2 cápsulas de 20 autopolinizaciones manuales y 1 de autopolinización espontánea que contrastan con la alta tasa de fructificación cuando las flores están libremente expuestas a los polinizadores. La reproducción sexual representa ventajas en el incremento de la capacidad de adaptación y en la eliminación de mutaciones desfavorables, sin embargo, implica la generación de atrayentes florales que generan un mayor gasto energético (Eguiarte et al. 1992).

\section{Agradecimientos}

Se reconoce al Consejo Nacional de Ciencia y Tecnología y a la Facultad de Ciencias de la Universidad Autónoma del Estado de México. También se le agradece a la Dra. Olivia Yanéz Ordóñez de la Facultad de Ciencias de la Universidad Nacional Autónoma de México, a Ellen B. Sperr de la Universidad de Ulm, Alemania y a María Guadalupe González Caballero de la Universidad Autónoma del Estado de México.

\section{Referencias}

Abad-Ibarra C, Navarro-Abad F \& Navarro A. 2008. El colibrí picolargo (Heliomaster constantii) en el Distrito Federal, México. Huitzil Revista de Ornitología Mexicana 9(001): 4-5.

Álvarez T. \& Álvarez-Castañeda S. 1996. Aspectos biológicos y ecológicos de los murciélagos de Ixtapan del Oro, México. En Contribution in Mammalogy: A Memorial volume Honoring Dr. J. Knox Jones. Jr. Museum Texas Tech University, pp. 169-182.

Arita H \& Martínez del Río C. 1990. Interacciones flormurciélago: Un enfoque zoocéntrico. Universidad Nacional Autónoma de México: Instituto de Biología.

Bojorges J. 2004. Riqueza de aves de la región Noreste de la Sierra Nevada, Estado de México. Acta Zoológica Mexicana 20(3): 15-29.

Buril M, Ribeiro F \& Alves M. 2008. Diversidade Palinológica das Convolvulaceae do Parque Nacional do Catimbau, Buíque, PE, Brasil. Acta Botánica 
Brasilica 22(4): 1163-1171.

Caballero-Martínez LA. 2007. Selección de alimento de Anoura geoffroyi (Chiroptera: Phyllostomidae) en Ixtapan del Oro, Estado de México. Tesis Maestría, Universidad Autónoma del Estado de México: Facultad de Ciencias.

Caballero-Martínez LA, Rivas IV \& Aguilera LI. 2009. Hábitos alimentarios de Anoura geoffroyi (CHIROPTERA: PHYLLOSTOMIDAE) en Ixtapan del Oro, Estado de México. Acta Zoológica Mexicana (n.s.) 25(1): 161-175

Canales D \& Altamirano T. 2004. Riqueza avifaunística del municipio de Isidro Fabela, Estado de México. Revista de Zoología Universidad Nacional Autónoma de México 015: 14-19.

Carranza EG.2001. Contribución al conocimiento de las plantas del género Ipomoea L. (Convolvulaceae) en el Estado de Guanajuato, México. Flora del Bajío y de Regiones Adyacentes. Fascículo complementario XVIII.

Carranza EG \& Murguía G. 1998. Una especie nueva de Ipomoea (Convolvulaceae), de los Estados de Guanajuato, Hidalgo y Querétaro, México. Acta Botánica Mexicana 45: 31-42.

Dieringer G, Cabrera L, Lara M, Loya L \& Reyes-Castillo P. 1999. Beetle pollination and floral thermogenicity in Magnolia tamaulipana (Magnoliaceae). International Journal of Plant Sciences 160: 64-71.

Dinerstein E. 1986. Reproductive ecology of fruit bats and the seasonality of fruit production in a Costa Rica cloud forest. Biotropica 18(4): 307-318.

Eguiarte L, Nuñez-Farfán J, Domínguez C \& Cordero C. 1992. Biología evolutiva de la reproducción en plantas. Ciencias 6: 69-86.

Faegri K \& van der Pijl L. 1979. The principles of pollination ecology. Oxford: Pergamon Press.

Fasola L, Bello M \& Guichón M. 2005. Uso de trampas de pelo y caracterización de los pelos de la ardilla de vientre rojo Callosciurus erythraeus. Mastozoología neotropical 12(1): 9-17.

Fix E \& Hodges J. 1951. Discriminatory analysis, nonparametric discrimination: Consistency properties. Technical Report 4, USAF School of Aviation Medicine, Randolph Field, Texas, Estados Unidos de Norteamérica. Republicado: 1989. International Statistical Review 57(3): 238-247.

Galleto L \& Bernardello G. 2004. Floral nectaries, nectar production dynamics and chemical composition in six Ipomoea Species (Convolvulaceae) in relation to pollinators. Annals of Botany 94: 269-280.

Gentry AH. 1995. Diversity and floristic composition of neotropical dry forests. En Seasonally Dry Tropical Forests (Bullock S, Mooney H \& Medina E, Eds.) Cambridge: Cambridge Univ. Press, pp. 146-194.

Girón MV. 1995. Análisis palinológico de la miel y la carga de polen colectada por Apis mellifera en el suroeste de Antioquia, Colombia. Boletín del Museo de Entomología de la Universidad del Valle 3: 35-54.

Goldblatt P, Bernhardt P \& Manning J. 2009. Adaptive radiation of the putridperianth: Ferraria (Iridaceae: Irideae) and its unusual pollinators. Plant Systematics and Evolution 278: 53-65.
Herrera G \& Martínez del Rio C. 1998. Pollen digestion by new world bats: effects of processing time and feeding habits. Ecology 79: 2828-2838.

Howell SN \& Webb S. 1995. A guide to the birds of Mexico and Northern Central America. Oxford: Oxford University Press.

Ibarra C, Iñiguez-Dávalos L \& Sánchez-Cordero V. 2005. Ecología de polinización de Stenocereus queretaroensis (CACTACEAE), un cactus columnar quiropterofílico en un bosque tropical seco de México. American Journal of Botany 92(3): 503-509.

Janzen DH. 1975. Ecology of plants in the tropics. Edward Arnold Publisher.

Kakeda K, Tsukada H \& Kowyama Y. 2000. A self-compatible mutant $S$ allele conferring a dominant negative effect on the functional $S$ allele in Ipomoea trifida. Sexual Plant Reproduction 13: 119-125.

Kunz TH. \& Whitaker JO. 1983. An evaluation of fecal analysis for determining food habits of insectivorous bats. Canadian Journal of Zoology 61: 1317-1321.

Kowyama Y, Takahasi H \& Muraoka K. 1994. Number, frequency and dominance relationships of S-alleles in diploid Ipomoea trifida. Heredity 73: 275-283.

Kowyama Y, Tsuchiya T \& Kakeda K. 2000. Sporophytic self-incompatibility in Ipomoea trifida, a close relative of sweet potato. Annals of Botany 85 (Suppl. A): 191-196.

Loyola RD, Antonini Y, Jacobi C \& Parentoni R. 2007. Disponibilidad de recursos florales en campos metalíferos: riqueza de especies, frecuencia de visitación y comportamiento de abejas. Bioikos, Campinas 21(1): 41-50.

Matuda E. 1966. Las Convolvuláceas de México. Gobierno del Estado de México; Dirección de Agricultura y Ganadería.

McDonald A. 1991. Origin and diversity of Mexican Convolvulaceae. Anales del Instituto de Biología de la Universidad Nacional Autónoma de México, Serie Botánica 62(1): 65-82.

McPherson G. 1981. Studies in Ipomoea (Convolvulaceae) I. The arborescens group. Ann. Missouri Bot. Gard. 68: 527-545

Medellín R, Arita H\& Sánchez O. 1997. Identificación de los murciélagos de México: Clave de campo. Asociación Mexicana de Mastozoología.

Miller RE, Rauscher MD \& Manos PS.1999. Phylogenetic systematics of Ipomoea (Convolvulaceae) based on ITS and waxy sequences. Systematic Botany 24(2): 209-227.

Miller RE, McDonald JA \& Manos PS. 2004. Systematics of Ipomoea subgenus Quamoclit (Convolvulaceae) based on ITS sequence data and a Bayesian phylogenetic analysis. American Journal of Botany 91(8): 1208-1218.

Miranda F \& Hernández X E. 1963. Los tipos de vegetación de México y su clasificación. Boletín de la sociedad Botánica de México 28: 29-179.

Muchhala N. 2006. The pollination biology of Burmeistera (Campanulaceae): specialization and syndromes. American Journal of Botany 93: 1081-1089.

Muchhala N.2007. Adaptive trade-off in floral morphology mediates specialization for flowers pollinated by 
bats and hummingbirds. American Naturalist 169(4): 494-504.

Muchhala N, Caiza A, Vizuete J \& Thomson J. 2008. A generalized pollination system in the tropics: bats, birds and Aphelandra acanthus. Annals of Botany 103: 1481-1487.

Muchhala, N. \& Jarrín-V P. 2002. Flower visitation by bats in cloud forests of Western Ecuador. Biotropica 34: 387-395.

Murguía SA, Márquez JG, Laguna GH \& Ponce MS. 1995. Estudio de frutos y semillas de Ipomoea teotitlanica McPherson (Convolvulaceae). Acta Botánica Mexicana 32: 69-77.

Nates-Parra G. 2005. Abejas silvestres y polinización. Manejo Integrado de Plagas y Agroecología (Costa Rica) 75: 7-20.

Navarijo OL \& Neri M. 2000. Lista avifaunística de San Francisco Oxtotilpan, Temascaltepec, Estado de México. Anales del Instituto de Biología Universidad Nacional Autónoma de México. Serie Zoología 71(1): 41-57.

Navarro L \& Medel R. 2009. Relationship between floral tube length and nectar robbing in Duranta erecta $\mathrm{L}$. (Verbenaceae). Biological Journal of the Linnean Society 96: 392-398.

Palacios-Chávez R, Arreguín-Sánchez ML \& QuirozGarcía DL. 1992. Polen de la familia Agavaceae de la Estación de Biología Chamela, Jalisco. Cactáceas y Suculentas Mexicanas 37: 87-92.

Parra-Tabla V. 2002. Ipomoea wolcottiana Rose (Convolvulaceae). En Historia natural de Chamela (Noguera F, Vega-Rivera J, García-Aldrete A \& Quesada-Avendaño M, Eds.). Universidad Nacional Autónoma de México: Instituto de Biología, pp. 568.

Peterson RT \& Chalif E. 2008. Aves de México: Guía de campo. México: Diana.

Potgieter CJ, Edwards TJ \& VanStaden J. 2009. Pollination of Plectranthus spp. (Lamiaceae) with sigmoid flowers in southern Africa. South African Journal of Botany 75: 646-659.

Proctor M, Yeo P \& Lack A. 1996. The Natural History of Pollination. Harper Collins Publisher. London, Glasgow.

Quesada M, Stoner K, Lobo J, Herrerías-Diego Y, Palacios-Guevara C, Munguía-Rosas M, Salazar K \& Rosas-Guerrero V. 2004. Effects of forest fragmentation on pollinator activity and consequences for plant reproductive success and mating patterns in bat-pollinated Bombacaceous trees. Biotropica 36(2): 131-138.
Rojas-Nossa S. 2007. Strategies of nectar extraction by Flowerpiercers (Aves: Diglossa and Diglossopis) and their effects on the pollination of high Andean plants. Ornitología Colombiana 5: 21-39.

Rzedowski J \& Rzedowski G. 1985. Flora fanerogámica del Valle de México. Vol. 2.México: Escuela Nacional de Ciencias Biológicas e Instituto de Ecología.

Sánchez, T. \& Medellín R. 2007. Food habits of the threatened bat Leptonycteris nivalis (Chiroptera: Phyllostomidae) in a mating roost in Mexico. Journal of Natural History 41: 25, 1753-1764.

Schemske D \& Bradshaw H. 1999. Pollinator preference and the evolution of floral traits in monkeyflowers (Mimulus). Proc. Natl. Acad. Sci. USA 96: 1191011915.

Sperr E, Caballero-Martínez LA, Medellín R \& Tschapka M. 2011. Seasonal changes in species composition, resource use and reproductive patterns within a guild of nectar-feeding bats in a west Mexican dry forest. Journal of Tropical Ecology 27: 133-145.

Stockwell EF. 2001. Morphology and flight manoeuvrality in new world leaf-nosed bats (Chiroptera: Phyllostomidae). Journal of Zoology (London) 254: 505514.

Telleria MC \& Daners G.2003. Pollen types in Southern new world Convolvulaceae and their taxonomic significance. Plant Systematics and Evolution 243: 99118.

Tovar A, Martínez Y \& Del Real L. 2009. Descripción de granos de polen de algunas plantas del municipio de Querétaro. Memorias de veranos de difusión, Universidad Autónoma de Querétaro, Octavo verano: 35-38.

Tschapka M \& Dressler S. 2002. Chiropterophily: on batflowers and flower bats. Royal Botanic Gardens 114125.

Tschapka M. 2004. Energy density patterns of nectar resources permit coexistence within a guild of Neotropical flower-visiting bats. Journal of Zoology 263: 7-21.

Waser NM, Price CL, Williams MV \& Ollerton NM. 1996. Generalization in pollination systems, and why it matters. Ecology 77: 1043-1060.

Wiens, D, Calvin, CL, Wilson CA, Davern Cl, Frank C \& Seavey SR. 1987. Reproductive success, spontaneous embryo abortion, and genetic load in flowering plants. Oecologia 71: 501-509.

Zar JH. 1996. Biostatistical Analysis. Nueva Jersey: Prentice Hall. 